\title{
Power optimization under brightness and communication requirements for visible light communication based on MacAdam ellipse
}

\author{
Shun $\mathrm{Lou}^{1}$, Chen Gong ${ }^{1 *}$, Nan $\mathrm{Wu}^{1}$, Zhengyuan $\mathrm{Xu}^{1,2}$ \\ 1. Key Laboratory of Wireless-Optical Communications, Chinese Academy of Sciences, \\ School of Information Science and Technology, University of Science and Technology of China, Hefei 230027, China \\ 2. Shenzhen Graduate School, Tsinghua University, Shenzhen 518055, China \\ * Corresponding author, Email: cgong821@ustc.edu.cn
}

\begin{abstract}
Data transmission with RGB LEDs is attracting significant research interest in VLC (Visible Light Communication). We consider the power optimization under brightness and communication requirements for RGB LEDs, and model the color constraint using the MacAdam ellipse instead of a fixed point in the chromaticity diagram. Then an optimization problem is formulated to determine the transmission power consumption and the corresponding coordinates in the chromaticity diagram. We propose a novel two-step algorithm to solve the optimization problem with lower implementation complexity. Numerical results show that the proposed approach shows the same performance as the optimal solution using a brute-force method, and requires the lower power consumption using MacAdam ellipse instead of merely a fixed point in the chromaticity diagram.
\end{abstract}

Keywords: VLC, power optimization, RGB LEDs, chromaticity diagram, MacAdam ellipse, cone programming

Citation: S. Lou, C. Gong, N. Wu, et al. Power optimization under brightness and communication requirements for visible light communication based on MacAdam ellipse [J]. Journal of communications and information networks, 2017, 2(4): 28-35.

\section{Introduction}

In recent years, VLC is attracting more and more attention $^{[1]}$, and has been regarded as a potential candidate for the future short-range communication with high speed ${ }^{[2]}$. A common realization is to use a white LED as the transmitter ${ }^{[3]}$, due to its long service life, less heat emission and low power con- sumption. Two common approaches are used to produce white light illumination LEDs. One is to stimulate the yellow fluorescent powder through a blue LED. The other is to mix red, green and blue light to produce white light that is stimulated by three wavelength fluorescent powder. The latter one is preferred for high communication rate requirements, due to the larger bandwidth for RGB pure

Manuscript received Jun. 04, 2017; accepted Jul. 25, 2017

This work was supported by National Key Basic Research Program of China (No. 2013CB329201), Key Program of National Natural Science Foundation of China (No. 61631018), Key Research Program of Frontier Sciences of CAS (No. QYZDY-SSWJSC003), Key Project in Science and Technology of Guangdong Province (No. 2014B010119001), Shenzhen Peacock Plan (No. 1108170036003286), and the Fundamental Research Funds for the Central Universities. 
light $\operatorname{LEDs}^{[4,5]}$. An LED can be used for both illumination and data transmission simultaneously ${ }^{[6]}$, and the standards on these topics have been proposed in IEEE 802.15.7 $7^{[7]}$. Appropriate modulation schemes ${ }^{[8-10]}$ and capacity analysis ${ }^{[11,12]}$ have been investigated for dimming control. On the other hand, the illumination quality must be guaranteed for VLC with RGB LEDs, including brightness and color requirements.

Recently, energy efficient joint brightness control and data transmission using white LEDs have been proposed in Refs. [13,14]. The work in Ref. [15] analyzes the energy consumption under lighting constraints with RGB LEDs for VLC. However, the color constraint in the chromaticity diagram is regarded as a fixed point. Multi-user sum-rate optimization with brightness control for multiple LEDs has been investigated in Ref. [16]. The power optimization needs to be investigated under brightness and communication requirements based on MacAdam ellipse, where certain illumination tolerance is incorporated.

In this paper, we investigate the power consumption optimization of RGB colors while meeting the brightness and communication requirements simultaneously. We model the color constraint using a MacAdam ellipse instead of a fixed point in the chromaticity diagram ${ }^{[17]}$. We formulate an optimization problem to determinate the system parameters, and propose a novel two-step algorithm to solve the problem. Numerical results show that the proposed approach can lead to an optimal solution with reduced computational complexity, as well as lower power consumption by using MacAdam ellipse instead of merely a fixed point in the chromaticity diagram.

The remainder of this paper is organized as follows. Section 2 presents the system model and characterizes the chromaticity diagram, MacAdam ellipse, brightness and communication requirements in a VLC system. Section 3 formulates an optimization problem to minimize the total transmission power consumption, and then proposes a novel two-step algorithm to solve the problem efficiently. Numerical results are shown in section 4 , and the conclusions are presented in section 5 .

\section{System model}

\subsection{Chromaticity diagram}

The human eye is a sensory organ, which can be regarded as a detector that enables the perception of electromagnetic radiation. A normal human eye has three kinds of cones - short (S), medium (M) and long (L), which can perceive different peak wavelengths. Optical stimulus with a SPD (Spectral Power Distribution) $\varphi(\lambda)$ will induce optical sensation of the different cones, which are called tristimulus values $X, Y$ and $Z$, as given by

$$
\begin{aligned}
& X=\int \varphi(\lambda) \alpha_{1}(\lambda) \mathrm{d} \lambda, \\
& Y=\int \varphi(\lambda) \alpha_{2}(\lambda) \mathrm{d} \lambda, \\
& Z=\int \varphi(\lambda) \alpha_{3}(\lambda) \mathrm{d} \lambda,
\end{aligned}
$$

where $\alpha_{1}(\lambda), \alpha_{2}(\lambda)$ and $\alpha_{3}(\lambda)$ denote the spectral responses of the three kinds of cones. Thus it is possible for multiple different SPDs to project onto the same point and produce the same sensations, which is called metameric equivalence ${ }^{[18]}$.

Assume that the SPD $\varphi(\lambda)$ of an RGB LED lamp is given by $\varphi(\lambda)=\sum_{i=1}^{3} \bar{P}_{i} S_{i}(\lambda)$, where $S_{1}(\lambda), S_{2}(\lambda)$ and $S_{3}(\lambda)$ denote the PSDs of red, green and blue colors, respectively, and $\bar{P}_{1}, \bar{P}_{2}$ and $\bar{P}_{3}$ denote the average transmission power of red, green and blue colors, respectively. The tristimulus value $X$ can be reformulated as

$$
\begin{aligned}
X & =\int \sum_{i=1}^{3} \bar{P}_{i} S_{i}(\lambda) \alpha_{1}(\lambda) \mathrm{d} \lambda \\
& =\sum_{i=1}^{3} \bar{P}_{i} \int S_{i}(\lambda) \alpha_{1}(\lambda) \mathrm{d} \lambda, \\
& =\sum_{i=1}^{3} \bar{P}_{i} g_{1 i} .
\end{aligned}
$$

and

$$
Y=\sum_{i=1}^{3} \bar{P}_{i} g_{2 i}, \quad Z=\sum_{i=1}^{3} \bar{P}_{i} g_{3 i},
$$


where $g_{i j}=\int S_{j}(\lambda) \alpha_{i}(\lambda) \mathrm{d} \lambda$ for $1 \leqslant i, j \leqslant 3$. Define $\boldsymbol{C}^{\prime} \triangleq[X, Y, Z]^{\mathrm{T}}$ and $\overline{\boldsymbol{P}} \triangleq\left[\bar{P}_{1}, \bar{P}_{2}, \bar{P}_{3}\right]^{\mathrm{T}}$. The relation between the average transmission power and tristimulus values can be expressed as

$$
C^{\prime}=G \bar{P},
$$

where $\boldsymbol{G} \triangleq\left[g_{i j}\right]_{1 \leqslant i, j \leqslant 3}$ denotes the transmission matrix.

Through the color matching experiment, CIE set up a series of color measurement and calculation methods, which were combined in CIE standard colorimetric system. In CIE 1931 chromaticity diagram, the point $(x, y)$ is given by

$$
\begin{aligned}
& x=\frac{X}{X+Y+Z}, \\
& y=\frac{Y}{X+Y+Z}, \\
& z=\frac{Z}{X+Y+Z} .
\end{aligned}
$$

Defining $C \triangleq[x, y, z]^{\mathrm{T}}$, we can express the relation between the average transmission power and the point in the chromaticity diagram as

$$
\boldsymbol{C}=k \boldsymbol{C}^{\prime}
$$

where $k=1 /(X+Y+Z)=1 /\left(\mathbf{1}^{\mathrm{T}}(\boldsymbol{G} \overline{\boldsymbol{P}})\right)$ denotes the normalized coefficient, and $\mathbf{1}=[1,1,1]^{\mathrm{T}}$. We notice that matrix $\boldsymbol{G}$ does not vary with parameters due to the invariable distribution of PSDs and responses of cones. According to Eqs. (4) and (6), we can find a matrix $\boldsymbol{H}$ such that

$$
\overline{\boldsymbol{P}}=\kappa \boldsymbol{G}^{-1} \boldsymbol{C}=\kappa \boldsymbol{H d},
$$

where $\kappa=1 / k=\mathbf{1}^{\mathrm{T}}(\boldsymbol{G} \overline{\boldsymbol{P}})$ and $\boldsymbol{d} \triangleq[x, y, 1]^{\mathrm{T}}$.

\subsection{MacAdam ellipse}

In CIE 1931 chromaticity diagram, every point denotes a certain chromaticity, i.e., the point $(0.33,0.33)$ denotes white light. However, as the coordinates of chromaticity vary within a certain range, chromaticity change can not be perceived due to the human eye limitation. This is called chromaticity tolerance, which can be characterized by MacAdam ellipse $^{[17]}$. As shown in Fig. 1, the chromaticity tolerance is not the same size, for example, the blue part of the tolerance is small, while the green part of that is big. The direction of each chromaticity tolerance is not the same either. We notice that MacAdam ellipse can be obtained by translation and rotation transformation from the standard ellipse, as given by

$$
\begin{aligned}
& \left(\frac{x^{\prime}}{a}\right)^{2}+\left(\frac{y^{\prime}}{b}\right)^{2}=1, \\
& x^{\prime}=\left(x-x_{0}\right) \cos \theta+\left(y-y_{0}\right) \sin \theta, \\
& y^{\prime}=\left(x-x_{0}\right) \sin \theta-\left(y-y_{0}\right) \cos \theta,
\end{aligned}
$$

where $\theta$ denotes the rotation angle between the major axis of the ellipse and $x$ axis, $a$ and $b$ denote the major and minor semi-axis of one step MacAdam ellipse, respectively, and $x_{0}$ and $y_{0}$ denote horizontal and vertical coordinates of the objective chromaticity. The matrix expression for the constraint of chromaticity tolerance can be given by

$$
\boldsymbol{d}^{\mathrm{T}} \boldsymbol{Q} \boldsymbol{d} \leqslant 1
$$

where $\boldsymbol{Q}$ can be obtained by $x_{0}, y_{0}, a, b$ and $\theta$. Note that the matrix $\boldsymbol{Q}$ is positive semi-definite for $\boldsymbol{d}$. The major and minor semi-axes of $l$-step MacAdam ellipse are $l$ times of $a$ and $b$, given by $l a$ and $l b$, respectively.

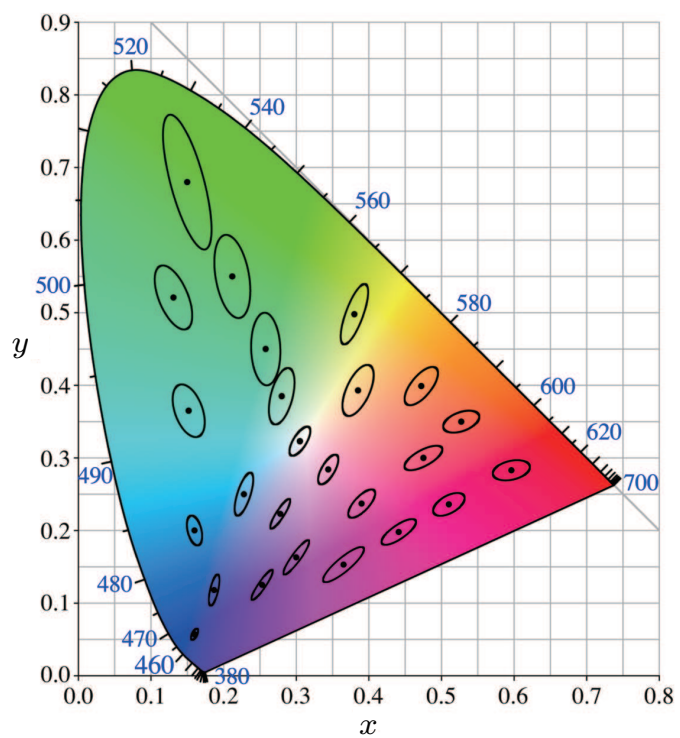

Figure 1 MacAdam ellipse (10 times the actual ellipse) plotted on the CIE 1931 chromaticity diagram 


\subsection{Brightness requirement}

In colorimetry, we use luminous flux $\phi$ to describe radiative flux induced by human visual strength, as given by

$$
\phi=K_{m} \int \varphi(\lambda) y(\lambda) \mathrm{d} \lambda=K_{m} Y
$$

where $K_{m}=683 \mathrm{~lm} / \mathrm{w}$. According to Eqs. (4), (6) and (7), we have the following

$$
Y=\boldsymbol{g}^{\mathrm{T}} \overline{\boldsymbol{P}}=\kappa y
$$

where $\boldsymbol{g} \triangleq\left[g_{21}, g_{22}, g_{23}\right]^{\mathrm{T}}$. The illumination $E$ denotes the illuminated surface flux per unit area, given by $E=\phi \xi$, where $\xi$ denotes the optical power gain. According to Eqs. (10) and (11), we have the following results, on the illumination $E$,

$$
E=K_{m} \boldsymbol{g}^{\mathrm{T}} \overline{\boldsymbol{P}} \xi=K_{m} \kappa y \xi .
$$

Assume that $\mu$ denotes the minimum illumination requirement. The illumination constraint can be expressed as

$$
\boldsymbol{g}^{\mathrm{T}} \overline{\boldsymbol{P}} \geqslant \eta
$$

where $\eta=\mu /\left(K_{m} \xi\right)$.

\subsection{Communication requirement}

The instantaneous transmission power $P_{i}$ can be expressed as

$$
P_{i}=\bar{P}_{i}+p_{i}
$$

where $p_{i}$ is the instantaneous $\mathrm{AC}$ component. The communication requirement can be denoted by the SINR (Signal to Interference Plus Noise Ratio) at the receiver. Then the SINR at the receiver $k$ can be expressed as

$$
S I N R_{k}=\frac{\left(\rho T_{i}^{k}\right)^{2} \mathbb{E}\left[p_{i}^{2}\right]}{\sum_{j \neq i}\left(\rho T_{j}^{k}\right)^{2} \mathbb{E}\left[p_{j}^{2}\right]+\sigma^{2}} \geqslant \Gamma_{k},
$$

where $\rho$ is the detector responsivity, $T_{i}^{k}$ is the link gain of color $i$ to user $k, \sigma^{2}$ is the noise variance and $\Gamma_{k}$ denotes the minimum SINR requirement of receiver $k$. We notice that for a moderate SINR regime, the interference term in Eq. (15) can be neglected, because the PSDs of RGB colors are almost not overlapping and the filter passband at the receiver is separated. Then we can get the following

$$
\mathbb{E}\left[p_{i}^{2}\right] \geqslant \max _{k} \frac{\sigma^{2} \Gamma_{k}}{\left(\rho T_{i}^{k}\right)^{2}} .
$$

When the modulation mode is determined, we can get the relationship $\mathbb{E}\left[p_{i}^{2}\right]=\beta_{i}\left(\max \left|p_{i}\right|\right)^{2}$, where $\beta_{i}$ denotes coefficient of modulation mode. The instantaneous transmission power $P_{i}$ is limited due to the LED nonlinearity, given by $P_{i}^{\min } \leqslant P_{i} \leqslant P_{i}^{\max }$. Letting

$$
\tau_{i}=\max _{k} \frac{\sigma^{2} \Gamma_{k}}{\left(\rho T_{i}^{k}\right)^{2}},
$$

we can get the average transmission power constraint according to Eqs. (14) and (16), given by

$$
\gamma^{\min } \leqslant \overline{\boldsymbol{P}} \leqslant \gamma^{\max },
$$

where $\boldsymbol{\gamma}^{\min }=\left[\gamma_{i}^{\min }\right]_{1 \leqslant i \leqslant 3}^{\mathrm{T}}$ and $\boldsymbol{\gamma}^{\max }=\left[\gamma_{i}^{\max }\right]_{1 \leqslant i \leqslant 3}^{\mathrm{T}}$, $\gamma_{i}^{\min }=P_{i}^{\min }+\sqrt{\tau_{i} / \beta_{i}}$ and $\gamma_{i}^{\max }=P_{i}^{\max }-\sqrt{\tau_{i} / \beta_{i}}$ denote the minimum and maximum permitted average transmission power of different colors, respectively.

\section{System design}

\subsection{Problem formulation}

In this section, we investigate the power consumption optimization under brightness and communication requirements for RGB-LED-based VLC. The optimization problem is formulated to determine the optical power distribution of RGB LEDs and the point coordination in the chromaticity diagram, as given by

$$
\begin{aligned}
\left(\mathcal{P}_{0}\right): \min _{\bar{P}_{i}, x, y} & \mathbf{1}^{\mathrm{T}} \overline{\boldsymbol{P}} \\
\text { s.t. } & \overline{\boldsymbol{P}}=\kappa \boldsymbol{H} \boldsymbol{d} ; \boldsymbol{g}^{\mathrm{T}} \overline{\boldsymbol{P}} \geqslant \eta ; \\
& \boldsymbol{\gamma}^{\min } \leqslant \overline{\boldsymbol{P}} \leqslant \boldsymbol{\gamma}^{\max } ; \boldsymbol{d}^{\mathrm{T}} \boldsymbol{Q} \boldsymbol{d} \leqslant 1,
\end{aligned}
$$

where $\kappa=\mathbf{1}^{\mathrm{T}}(\boldsymbol{G} \overline{\boldsymbol{P}})$. We notice that $\boldsymbol{d}=\boldsymbol{H}^{-1} \overline{\boldsymbol{P}} / \kappa$ according to the equality constraint, then the MacAdam ellipse can be expressed as

$$
\overline{\boldsymbol{P}}^{\mathrm{T}}\left(\boldsymbol{H}^{-1}\right)^{\mathrm{T}} \boldsymbol{Q}\left(\boldsymbol{H}^{-1}\right) \overline{\boldsymbol{P}} \leqslant \kappa^{2} .
$$

We notice that matrix $\boldsymbol{Q}$ is positive semi-definite, then $\left(\boldsymbol{H}^{-1}\right)^{\mathrm{T}} \boldsymbol{Q}\left(\boldsymbol{H}^{-1}\right)$ can be transmitted as $\boldsymbol{U}^{\mathrm{T}} \boldsymbol{U}$. 
Thus the optimization problem can be reformulated as

$$
\begin{aligned}
\left(\mathcal{P}_{1}\right): & \min _{\bar{P}_{i}, x, y} \mathbf{1}^{\mathrm{T}} \overline{\boldsymbol{P}} \\
\text { s.t. } & (\boldsymbol{U} \overline{\boldsymbol{P}})^{\mathrm{T}} \boldsymbol{U} \overline{\boldsymbol{P}} \leqslant \kappa^{2} ; \boldsymbol{g}^{\mathrm{T}} \overline{\boldsymbol{P}} \geqslant \eta ; \\
& \boldsymbol{\gamma}^{\mathrm{min}} \leqslant \overline{\boldsymbol{P}} \leqslant \boldsymbol{\gamma}^{\max } .
\end{aligned}
$$

The optimization problem $\left(\mathcal{P}_{1}\right)$ can be regarded as the cone programming, which could be solved by CVX toolbox in MATLAB. However, the solution may require large number of iterations to obtain the optimal solution. To reduce the computational complexity, we propose a novel two-step algorithm to solve the optimization problem efficiently, which is elaborated as follows.

\subsection{The first step}

We notice that the optimization problem $\left(\mathcal{P}_{0}\right)$ can be regarded as linear programming once $\boldsymbol{d}$ is fixed. In other words, the feasible region of MacAdam ellipse has become a fixed point in the chromaticity diagram. Then the optimization problem can be expressed as

$$
\begin{array}{ll}
\left(\mathcal{P}_{2}\right): & \min _{\bar{P}_{i}} \boldsymbol{1}^{\mathrm{T}} \overline{\boldsymbol{P}} \\
\text { s.t. } & \overline{\boldsymbol{P}}=\kappa \boldsymbol{H} \boldsymbol{d} ; \boldsymbol{g}^{\mathrm{T}} \overline{\boldsymbol{P}} \geqslant \eta ; \\
& \boldsymbol{\gamma}^{\min } \leqslant \overline{\boldsymbol{P}} \leqslant \boldsymbol{\gamma}^{\max } .
\end{array}
$$

It is generally known that the solution of linear programming is the intersection of feasible regions. The first equality constraint shows that $\overline{\boldsymbol{P}}$ can be regarded as a scaled version of a fixed vector due to the coefficient $\kappa$. Therefore, the solution to problem $\left(\mathcal{P}_{2}\right)$ is the scaled version of vector $\boldsymbol{H} \boldsymbol{d}$ in the intersections of two regions characterized by the "brightness requirement" and "communication requirement" constraints as the first and second inequality constraints, respectively.

\subsection{1 "Communication requirement" equal- ity}

We first consider the "communication requirement" achieving equality. Since there are six equations in this constraint, without loss of generality, we assume that $\bar{P}_{1}=\gamma_{1}^{\max }$. According to Eq. (7), the coefficient $\kappa$ can be expressed as $\kappa=\gamma_{1}^{\max } /\left(h_{11} x+h_{12} y+h_{13}\right)$. Then the average transmission power $\bar{P}_{i}$ is given by $\bar{P}_{i}=\gamma_{1}^{\max }\left(h_{i 1} x+h_{i 2} y+h_{i 3}\right) /\left(h_{11} x+h_{12} y+h_{13}\right)$. The variables $\bar{P}_{i}$ can be removed in the optimization problem $\left(\mathcal{P}_{2}\right)$. Then the objective function for total average power consumption is equivalent to the following objective function,

$$
\frac{\left(h_{21}+h_{31}\right) x+\left(h_{22}+h_{32}\right) y+\left(h_{23}+h_{33}\right)}{h_{11} x+h_{12} y+h_{13}} .
$$

We eliminate the variable $x$ in the numerator, and expression (22) can be reformulated as

$$
\frac{p y+q}{h_{11} x+h_{12} y+h_{13}}+\frac{h_{21}+h_{31}}{h_{11}},
$$

where $p$ and $q$ can be denoted by elements $h_{i j}$ in the matrix $\boldsymbol{H}$. We notice that $\boldsymbol{H}$ does not vary with parameters, due to the invariable matrix $\boldsymbol{G}$. Matrix $\boldsymbol{H}$ can be written as follows,

$$
\boldsymbol{H}=\left(\begin{array}{ccc}
2.6420 & -1.9482 & -0.1536 \\
-0.7278 & 1.8769 & -0.0808 \\
-0.6646 & -0.6681 & 0.6657
\end{array}\right) .
$$

It is seen the last item in expression (23) is always a negative constant. Therefore, the first item in expression (23) must be positive, since the whole equation is positive. Then we could take the inverse of the first item directly. We eliminate variable $y$ from the numerator, and the final objective function of problem $\left(\mathcal{P}_{2}\right)$ can be expressed as

$$
\frac{u}{v} \cdot \frac{x+m}{y+n},
$$

where $u, v, m$ and $n$ can be readily obtained from expression $(23)$. Then the optimization problem $\left(\mathcal{P}_{0}\right)$ can be reformulated as

$$
\begin{aligned}
\left(\mathcal{P}_{3}\right): \max _{x, y} & \frac{u}{v} \cdot \frac{x+m}{y+n} \\
\text { s.t. } & \boldsymbol{L} \boldsymbol{d} \leqslant \mathbf{0} ; \quad \boldsymbol{d}^{\mathrm{T}} \boldsymbol{Q} \boldsymbol{d} \leqslant 1,
\end{aligned}
$$

where $\boldsymbol{L} \in \mathbb{R}^{5 \times 3}$ can be obtained by linear constraints from problem $\left(\mathcal{P}_{2}\right)$.

\subsection{2 "Brightness requirement" equality}

In this part, we consider "brightness requirement" constraint achieving equality, which is $\boldsymbol{g}^{\mathrm{T}} \overline{\boldsymbol{P}}=\eta$. Then we can have $\kappa=\eta / y$ based on Eq. (11). Ac- 
cording to Eq. (7), the average transmission power $\bar{P}_{i}$ can be expressed as $\bar{P}_{i}=\eta\left(h_{i 1} x+h_{i 2} y+h_{i 3}\right) / y$. We remove the variables $\bar{P}_{i}$ of the optimization problem $\left(\mathcal{P}_{2}\right)$, then the optimization problem $\left(\mathcal{P}_{0}\right)$ can be reformulated as

$$
\begin{array}{rl}
\left(\mathcal{P}_{4}\right): \min _{x, y} & s . \frac{x+t}{y} \\
\text { s.t. } & \boldsymbol{B} \boldsymbol{d} \leqslant \mathbf{0} ; \quad \boldsymbol{d}^{\mathrm{T}} \boldsymbol{Q} \boldsymbol{d} \leqslant 1,
\end{array}
$$

where $s$ and $t$ can be expressed by elements $h_{i j}$ in matrix $\boldsymbol{H}$, and $\boldsymbol{B} \in \mathbb{R}^{6 \times 3}$ can be obtained by linear constraints from problem $\left(\mathcal{P}_{2}\right)$.

\subsection{The second step}

Optimization problems $\left(\mathcal{P}_{3}\right)$ and $\left(\mathcal{P}_{4}\right)$ only determine the optimal point $(x, y)$ of the MacAdam ellipse in the chromaticity diagram subject to inequality constraints. For problem $\left(\mathcal{P}_{3}\right)$, it can be regarded as the maximum or minimum slope of the point $(-m,-n)$ to the feasible region, which is given by the MacAdam ellipse by line constraints. If the ellipse and lines do not intersect, the optimal solution is the tangent point from the point $(-m,-n)$ to the ellipse. While the feasible region is an ellipse cut by line constraints, the optimal solution is determined by the tangent point aforementioned or intersections between constraints. Note that the point aforementioned must be a feasible point meeting all constraints. Problem $\left(\mathcal{P}_{4}\right)$ can be solved similarly. The optimal solution to problem $\left(\mathcal{P}_{0}\right)$ is the point with the minimum objective function of all optimal solutions to problems $\left(\mathcal{P}_{3}\right)$ and $\left(\mathcal{P}_{4}\right)$.

The computational complexity of the proposed methods is mainly due to the second step, because we can obtain the explicit expression of $\bar{P}_{i}$ in the first step, then problems $\left(\mathcal{P}_{3}\right)$ and $\left(\mathcal{P}_{4}\right)$ can be formulated with the complexity of order $O(1)$. The optimal solution to problems $\left(\mathcal{P}_{3}\right)$ and $\left(\mathcal{P}_{4}\right)$ can be derived by investigating the slope from the point $(-m,-n)$, and the computational complexity is in the same order of the convex programming with only two variables, which is significantly lower than the interior point solution to the original problem.

\section{Numerical results}

Assume that an RGB LED lamp is employed as the transmitter to modulate signals. The cutoff wavelengths of the three filters at the receiver for red, green and blue colors are [300 nm, $520 \mathrm{~nm}$ ], [520 nm, $600 \mathrm{~nm}]$ and [600 nm, $800 \mathrm{~nm}$ ], respectively. The parameters of MacAdam ellipse are shown in Tab. 1 with different CCTs (Correlated Color Temperatures). $\Gamma_{k}$ is defined as $13.6 \mathrm{~dB}$, and $\sigma^{2}$ is set to $10^{-9} \mathrm{~W}$. Assume the channel DC gain $T_{i}^{k}=2 \times 10^{-4}$, the optical power gain $\xi=0.02$, the receiver responsivity $\rho=0.56$ and the modulation formulation coefficient $\beta_{i}=9 / 5$. The maximum and minimum instantaneous transmission power for each color are $P_{i}^{\max }=10 \mathrm{~W}$ and $P_{i}^{\min }=1 \mathrm{~W}$, respectively. $\mathrm{A}$ 7-step MacAdam ellipse is used based on America National Standard Institute (ANSI).

Table 1 MacAdam ellipse parameters under different CCTs

\begin{tabular}{ccccc}
\hline $\mathrm{CCT}$ & $\left(x_{0}, y_{0}\right)$ & $\theta$ & $a$ & $b$ \\
\hline $2700 \mathrm{~K}$ & $(0.459,0.412)$ & $53^{\circ} 42^{\prime}$ & 0.0027 & 0.0014 \\
$3000 \mathrm{~K}$ & $(0.440,0.403)$ & $53^{\circ} 13^{\prime}$ & 0.00278 & 0.00136 \\
$3500 \mathrm{~K}$ & $(0.411,0.393)$ & $54^{\circ} 00^{\prime}$ & 0.00309 & 0.00138 \\
$4000 \mathrm{~K}$ & $(0.380,0.380)$ & $53^{\circ} 43^{\prime}$ & 0.00313 & 0.00134 \\
$5000 \mathrm{~K}$ & $(0.346,0.359)$ & $59^{\circ} 37^{\prime}$ & 0.00274 & 0.00118 \\
\hline
\end{tabular}

Fig. 2 shows the transmission power of different colors versus illumination requirement ranging from 10 to 150 lux.

We notice that the proposed two-step method shows the same performance as the optimal solution solved by the CVX toolbox in MATLAB, with lower implementation complexity. We further notice that the proportion of the optical power distribution among three colors is relatively constant, due to the constraint of MacAdam ellipse. When an illumination requirement that is too large (i.e., more than 150 lux) results in an operating point outside the feasible power region, a feasible solution cannot be found.

We show the total transmission power consumption corresponding to different steps of MacAdam ellipse versus different illumination requirements, as 
shown in Fig. 3. We can see that the total transmission power first keeps constant and then increases with the illumination intensity. It implies that the illumination requirement is not the main constraint for low brightness. Moreover, the total transmission power decreases for larger steps of MacAdam ellipse, since the larger step of MacAdam ellipse denotes larger feasible range to select operating point in the chromaticity diagram. It also implies the lower power consumption using MacAdam ellipse instead of merely a fixed point in the chromaticity diagram. The total transmission power consumption versus illumination requirement for different CCTs is shown in Fig. 4. It is generally seen that the curve trend is similar for different CCTs.

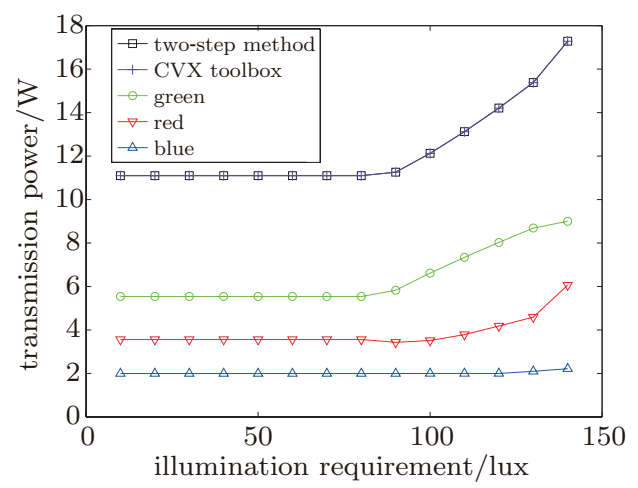

Figure 2 Transmission power versus illumination requirement under $3000 \mathrm{~K}$ and 7-step MacAdam ellipse

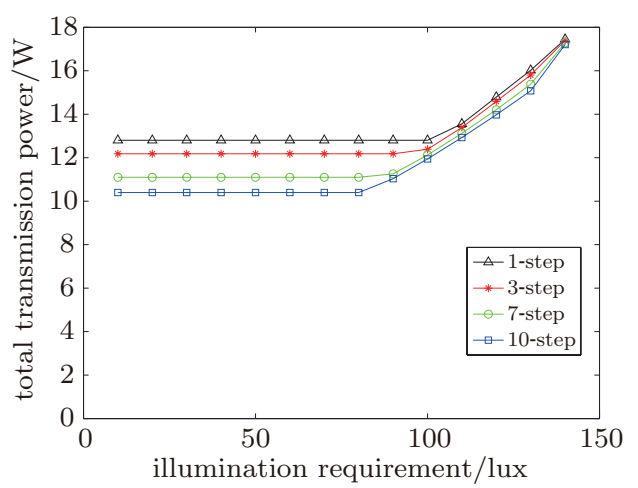

Figure 3 Total transmission power versus illumination requirement for different steps of MacAdam ellipse under $3000 \mathrm{~K}$

\section{Conclusions}

We investigated the problem of power optimization under brightness and communication requirements

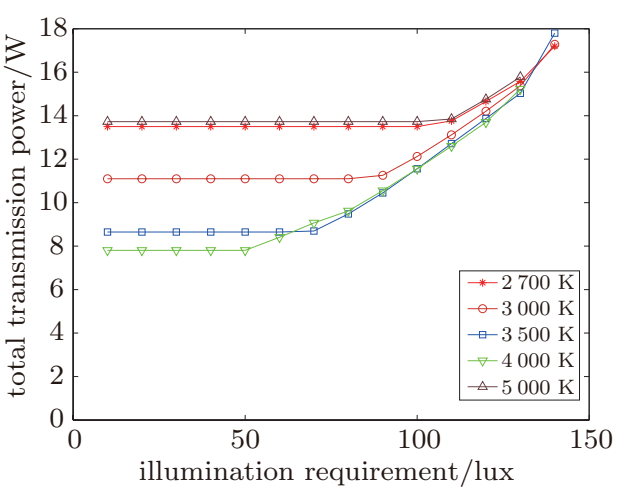

Figure 4 Total transmission power versus illumination requirement for different CCTs under 7-step MacAdam ellipse

for RGB LEDs with certain chromatic tolerance based on MacAdam Ellipse. We established a system model, and then formulated an optimization problem of minimizing the total transmission power under illumination and chromatic constraints. We proposed a novel two-step algorithm to solve the optimization problem effectively, which can lead to the optimal solution with reduced computational complexity. Numerical results imply the lower power consumption using MacAdam ellipse instead of merely a fixed point in the chromaticity diagram.

\section{References}

[1] A. Jovicic, J. Y. Li, T. Richardson. Visible light communication: opportunities, challenges and the path to market [J]. IEEE commun. mag., 2013, 51(12): 26-32.

[2] L. Grobe, A. Paraskevopoulos, J. Hilt, et al. High-speed visible light communication systems [J]. IEEE commun. mag., 2013, 51(12): 60-66.

[3] T. Komine, M. Nakagawa. Fundamental analysis for visible-light communication system using LED lights [J]. IEEE trans. consumer electron., 2004, 50(1): 100-107.

[4] G. Cossu, A. Khalid, P. Choudhury, et al. 3.4 Gbit/s visible optical wireless transmission based on RGB LED [J]. Opt. exp., 2012, 20(26): B501-B506.

[5] F. M. Wu, C. T. Lin, C. C. Wei, et al. 3.22-Gb/s WDM visible light communication of a single RGB LED employing carrier-less amplitude and phase modulation [C]//Optical Fiber Communication Conference, Anaheim, USA, 2013: OTh1G-4.

[6] J. Gancarz, H. Elgala, T. D. Little. Impact of lighting requirements on VLC systems [J]. IEEE commun. mag., 2013, 51(12): 34-41.

[7] S. Rajagopal, R. D. Roberts, S. K. Lim. IEEE 802.15.7 visible light communication: modulation schemes and 
dimming support [J]. IEEE commun. mag., 2012, 50(3): $72-82$.

[8] S. H. Lee, S. Y. Jung, J. K. Kwon. Modulation and coding for dimmable visible light communication $[\mathrm{J}]$. IEEE commun. mag., 2015, 53(2): 136-143.

[9] Q. Wang, Z. C. Wang, L. Dai. Asymmetrical hybrid optical OFDM for visible light communications with dimming control [J]. IEEE photon. technol. lett., 2015, 27(9): 974-977.

[10] Q. Wang, Z. C. Wang, L. L. Dai, et al. Dimmable visible light communications based on multilayer ACO-OFDM [J]. IEEE photon. j., 2016, 8(3): 1-11.

[11] J. B. Wang, Q. S. Hu, J. Wang, et al. Tight bounds on channel capacity for dimmable visible light communications [J]. J. lightw. technol., 2013, 31(23): 3771-3779.

[12] J. Y. Wang, J. B. Wang, N. Huang, et al. Capacity analysis for pulse amplitude modulated visible light communications with dimming control $[\mathrm{J}]$. J. opt. soc. am., 2014, 31(3): 561-568.

[13] I. Din, H. Kim. Energy-efficient brightness control and data transmission for visible light communication $[\mathrm{J}]$. IEEE photon. technol. lett., 2014, 26(8): 781-784.

[14] S. Shao, A. Khreishah, I. Khalil. Joint link scheduling and brightness control for greening VLC-based indoor access networks [J]. IEEE/OSA j. opt. commun. netw., 2016, 8(3): 148-161.

[15] C. Gong, S. B. Li, Q. Gao, et al. Power and rate optimization for visible light communication system with lighting constraints $[\mathrm{J}]$. IEEE trans. signal processing, 2015, 63(16): 4245-4256.

[16] R. Jiang, Z. C. Wang, Q. Wang, et al. Multi-user sumrate optimization for visible light communications with lighting constraints [J]. J. lightw. technol., 2016, 34(16): 3943-3952.

[17] D. L. Macadam. Visual sensitivities to color differences in daylight [J]. J. opt. soc. am., 1942, 32(5): 247-274.

[18] P. M. Butala, J. C. Chau, T. D. Little. Metameric modulation for diffuse visible light communications with constant ambient lighting [C]//International Workshop on Optical Wireless Communications, Pisa, 2012: 1-3.

\section{About the authors}

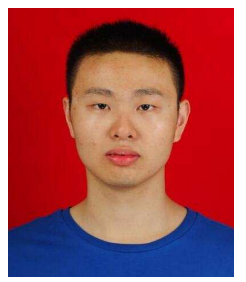

Shun Lou received the B.E. degree from University of Electronic Science and Technology of China, ChengDu, China, in 2015. He is now a M.S. candidate in University of Science and Technology of China (USTC). His research interest is optical wireless communications. (Email: loushun@mail.ustc.edu.cn)

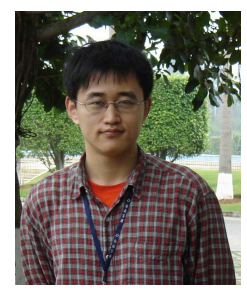

Chen Gong [corresponding author] received the B.S. degree in electrical engineering and mathematics (minor) from Shanghai Jiaotong University, Shanghai, China in 2005, M.S. degree in electrical engineering from Tsinghua University, Beijing, China in 2008, and Ph.D. degree in Columbia University, New York City, New York, USA in 2012. He was a senior systems engineer in Qualcomm Research San Diego, San Diego, Qualcomm Inc., between Apr. 2012 and Dec. 2013. Now he is on the faculty of University of Science and Technology of China (USTC). His research interest falls into the general area of wireless communications, optical wireless communications, and signal processing. $\mathrm{He}$ has been selected by the "Thousand Young Talents Program" of China Government in 2014. (Email: cgong821@ustc.edu.cn)

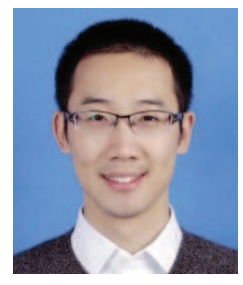

Nan Wu received his Ph.D. degree from New Jersey Institute of Technology in 2015. Now he is working as a postdoc researcher in University of Science and Technology of China. His research interest is optical wireless communications. (Email: nw27@ustc.edu.cn)

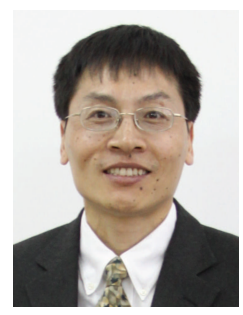

Zhengyuan $\mathbf{X u}$ received his B.S. and M.S. degrees from Tsinghua University, China, and Ph.D. degree from Stevens Institute of Technology, USA. He was with University of California, Riverside, during 1999-2010, where he became a full professor with tenure and also a founding director of UC-Light Center. In 2010, he was selected by the "Thousand Talents Program" of China and appointed as a professor at Tsinghua University. Since 2013, he has been a professor in Department of Electronic Engineering and Information Science, School of Information Science and Technology, University of Science and Technology of China. He is a founding director of Key Laboratory of Wireless-Optical Communications in Chinese Academy of Sciences, and chief scientist of the National Key Basic Research Program of China. His research focuses on wireless communication and networking, optical wireless communications, geolocation, and signal processing. He has published over 200 papers on journals and conferences. He was an associate editor and guest editor for different IEEE journals, and founding co-chair of IEEE Globecom Workshop on Optical Wireless Communications. He has delivered tutorials, keynote speeches and invited talks on optical wireless communications in various international conferences. (Email: xuzy@ustc.edu.cn) 